\title{
NON-STANDARD FORMAT CHAPTER
}

\section{A Historical Perspective on Bacterial Persistence}

\section{Running head:}

Historical Perspective on Bacterial Persistence

\section{Authors:}

Natalie Verstraeten, Wouter Knapen, Maarten Fauvart", Jan Michiels\#,*

\begin{abstract}
Affiliation
KU Leuven - University of Leuven, Centre of Microbial and Plant Genetics (CMPG), B-3001 Leuven, Belgium.
\end{abstract}

\#These authors contributed equally

${ }^{*}$ Corresponding author. E-mail: Jan.Michiels@biw.kuleuven.be 


\section{Summary/Abstract}

Bactericidal antibiotics quickly kill the majority of a bacterial population. However, a small fraction of cells typically survives through entering the so-called persister state. Persister cells are increasingly being viewed as a major cause of the recurrence of chronic infectious disease and could be an important factor in the emergence of antibiotic resistance. The phenomenon of persistence was first described in the 1940s, but remained poorly understood for decades afterwards. Only recently, a series of breakthrough discoveries has started to shed light on persister physiology and the molecular and genetic underpinnings of persister formation. We here provide an overview of the key studies that have paved the way for the current boom in persistence research, with a special focus on the technological and methodological advances that have enabled this progress.

\section{Key words}

Persisters, persistence, antibiotic tolerance, dormancy, antibiotics, review 


\section{The Early Days}

The first report on the survival of a small fraction of streptococci cells following treatment with penicillin dates from 1942 [1]. Two years later, Joseph Bigger established that addition of penicillin to staphylococci does not result in complete sterilization of all cells in a clonal population. One out of a million cells survived even prolonged treatment with antibiotics. He appropriately named the surviving cells persisters [2]. More recently, it was shown that in most bacterial species, the majority of cells is efficiently killed by relatively low concentrations of bactericidal antibiotics. However, killing shows a biphasic pattern and beyond a certain threshold, further increasing the concentration of the antibacterial does not result in complete clearing of the culture (Figure 1) [3].

\section{(FIGURE 1 HERE)}

For forty years following its discovery, the persistence phenomenon was largely neglected, at least by molecular geneticists. This was partly due to the fact that the clinical relevance of persister cells was not clear. In contrast, the threat posed by inherited antibiotic resistance was generally recognized, adding incentives to resistance research. The problem was compounded by technical challenges that inevitably accompany the study of a transient phenotype that is associated with only a very small fraction of cells.

A breakthrough discovery came in the early 1980s, from research carried out by Harris Moyed during a sabbatical leave in the lab of Alexander Tomasz [4]. Mutagenesis of $E$. coli populations with ethyl methanesulfonate (EMS) led to the identification of three high persistent (hip) mutants exhibiting 10- to 10,000-fold 
increased persister fractions upon incubation with penicillin [4,5]. Moyed's pioneering work led to the identification of two mutants hit in the hipA locus that up until now remains the best-studied persister gene [6-10]. Furthermore, because of their increased persister fraction, hipA mutants have frequently been used as a tool in persistence research. Crucially and for the first time, hipA mutants enabled the direct observation of persister cells. Using a combination of microfluidics and live cell microscopy, Nathalie Balaban recorded how persisters survived killing by antibiotics through dormancy and subsequent resuscitation [11]. In addition, the hipBA locus is a representative for other toxin-antitoxin (TA) loci that are now intensively studied in relation to persistence. TA modules consist of a stable toxin, typically targeting essential cellular functions, and an unstable antitoxin, which counteracts the activity of its cognate toxin $[12,13]$. TA systems were originally identified on plasmids, where they play a role in plasmid maintenance, yet a significant number of TA loci are chromosomally encoded and these have been implicated in persistence [14]. Examples include RelE [6], MqsR [15-17], TisB [18,19], MazF [20] and YafQ [21]. Interestingly, with the notable exception of Salmonella persisters residing within macrophage vacuoles [22], deletion of a single toxin generally does not affect persistence. This can partly be explained by redundancy of TA systems in most bacteria. Deletion of multiple TA systems, on the other hand, causes a decrease in $E$. coli persistence [23].

\section{The Rise of Persistence Research}

Following the discovery of hipA, persistence as a field of study steadily gained attention. This was partly due to the acknowledgement of its clinical significance (summarized by [24]). In 1944, Bigger already alluded to the role of persisters in the 
resuscitation of chronic infections [2]. Decades later, Kim Lewis postulated that persisters might contribute to the recalcitrance of biofilm infections $[25,26]$. This is of particular interest as biofilms are known to withstand antibiotic treatment, thereby causing chronic infections [27]. Subsequently, mathematical modelling demonstrated that persistence could extend the duration of antibiotic treatment, thereby causing treatment failure and promoting the emergence of resistance [28]. Finally, two studies have unambiguously demonstrated that prolonged antimicrobial therapy selects for high-persistent strains of Candida albicans during candidiasis and of Pseudomonas aeruginosa during cystic fibrosis lung infections [29,30]. In addition, the role of persister cells in the development of resistance is becoming increasingly clear [31]. Apart from providing incentives to further intensify persistence research, these findings also promoted the search for anti-persister therapies. At present, several strategies have been described, but their in vivo effectiveness remains to be investigated. Examples include the use of resonant activation [32], electrochemical currents [33], cadaverine [34], metabolites [35,36], antimicrobial peptides [37], brominated furanones [38-41] and activated CIpP [42] (summarized by [43]).

Apart from increased interest due to the clinical importance of persistence, the development of novel techniques also caused persistence research to boom. An overview of these novel techniques is provided below.

\section{$\underline{\text { Screening approaches }}$}

Over the years, several screening procedures have been developed that led to the identification of persister genes. In a first approach, a non-redundant $E$. coli knockout library was screened for mutants with altered persistence [44]. Persister cells of individual mutants were quantified by treating a stationary-phase culture with 
ofloxacin and plating the surviving cells on agar medium containing amdinocillin. As the number of spontaneous amdinocillin-resistant mutants is a fraction of the original number of cells, this obviates the need for dilution steps and greatly reduces the laborious task of screening several thousands of strains.

A second screening approach employed a $P$. aeruginosa plasposon knock-out library. Individual mutants were grown until stationary phase and treated with either ofloxacin to kill non-persister cells or water, the latter serving as a control. Subsequently, samples were diluted and incubated in an automated plate reader (Bioscreen C, Oy Growth Curves Ab Ltd), allowing the optical density of 200 samples to be measured simultaneously as a function of time. Given the linear relationship between the number of cells in an inoculum and the lag phase, this allowed for the selection of mutants displaying altered persister levels [45].

Both screenings led to the identification of a number of interesting persister genes including some global regulators. In addition, not a single mutant lacking persisters was identified. As a general conclusion, these screenings therefore provided evidence pointing to the multiplicity of persister formation mechanisms.

In a final approach, a random overexpression library was generated in E. coli. Cells from the recombinant library were pooled and logarithmically growing cultures of library clones were exposed to multiple rounds of exposure to ampicillin. This led to the enrichment of mutants with increased probability of persister formation and ultimately to the identification of $g / p D$ as a genuine persister gene [46].

\section{Single-cell studies}

As persistence is a phenotypic trait expressed in only a subfraction of a population, advances in single-cell research signified an era of vast new possibilities. First used 
by the Balaban group [11], transparent microfluidic devices proved instrumental for microscopic examination of persister cells [47-49]. The strength of this technique lies in the possibility to monitor individual cells for prolonged periods of time while adapting growth conditions. For example, normal growth conditions can be alternated with antibiotic treatments in order to kill non-persister cells. This allows to pinpoint persister cells surviving treatment. Subsequently, the history of persister cells can be traced back through the recorded images. Several studies have used this technique to demonstrate preexisting heterogeneity in bacterial populations [11], to characterize the dormant state of single persister [47], to monitor persistence formation following administration of indole [48] and to correlate high TA expression to cessation of growth [49].

Also developed by the Balaban group, a colony-appearance assay was elaborated to quantify single-cell persister lag phases [50]. Experiments demonstrated that a threshold concentration of toxin molecules is required for induction of persistence.

A major drawback of microfluidic devices, or more precisely of microscopy, is the limited number of cells that can be studied simultaneously. This can be circumvented by using flow cytometry, allowing thousands or even millions of cells to be evaluated in a high-throughput manner. A shortcoming of this technique is the inability to continuously monitor individual cells over time. Nonetheless, flow cytometry has been successfully used to study the kinetics of persister awakening [51]. In addition, while Bigger postulated that persisters are in a dormant, non-dividing state [2], flow cytometry has been used to demonstrate that dormancy is not a requirement for entry into the persister state [52]. Finally, a recent study performed by the Holden group showed how to characterize the dynamics of intracellular bacterial replication at the single cell level. They used a fluorescence dilution technique to quantify the number 
of replication cycles of internalized Salmonella [53]. This showed the existence of different Salmonella subpopulations in bone marrow-derived macrophages including a non-replicating but metabolically active subpopulation, comprising the persister cells, possibly capable of resuming growth and causing relapsing infections [22]. Similarly, the Bumann group exploited a DsRed variant called TIMER ${ }^{\text {bac }}$, which spontaneously changes color from green to green/orange over time, as a dynamic growth rate reporter to identify persister cells in vivo [54].

\section{$\underline{\text { Transcriptomics }}$}

Insight into global transcriptional changes in persister cells came from several elegant studies by the Lewis group. To enrich for persisters, all three approaches conveniently employed the metabolic inactivity of these cells. In the first report, logarithmically growing populations of the high-persistence E. coli mutant hipA7 [4] were treated with ampicillin, thereby lysing non-persister cells. Isolated RNA was enriched for mRNA, labeled and hybridized to E. coli GeneChips [6]. Similarly, gene expression profiling of persisters was performed after treating an exponentially growing population of Mycobacterium tuberculosis with D-cycloserine and collecting surviving persister cells by centrifugation. Transcriptome analysis was performed by microarray hybridization [55]. The third study followed a slightly different approach. It was based on the assumption that persisters are dormant cells with low levels of protein synthesis and corresponding low levels of rRNA transcription. E. coli persister cells were isolated by linking the $r r n B$ promoter to a gene encoding an unstable fluorescent protein. In so doing, persister cells are dim as compared to normal cells in the population, which allows for the isolation of persisters using Fluorescence 
Activated Cell Sorting (FACS). cDNA was prepared from purified RNA and hybridized to spotted E. coli DNA microarrays [56].

Based on the studies cited above, stress response pathways as well as TA loci were shown to be highly expressed in isolated persister cells. On the other hand, biosynthetic functions including energy production were downregulated $[6,55,56]$.

\section{Experimental evolution}

The use of experimental evolution for elucidating antibacterial resistance mechanisms is a widely used method. A recent study by the Balaban group used this technique for enriching a population with persisters by repeated exposure of a bacterial population to high concentrations of antibiotics. This resulted in evolved strains showing very high persister fractions caused by fixed specific genetic mutations. The increased survival appeared to be the result of an adjustment in the single cell lag-time distribution, which was correlated with the extent of the antibiotic exposure interval [57]. They implemented the ScanLag method, which allows the simultaneous measurement of lag times of hundreds of cells [58]. These findings resulted in a new theory regarding persister cells and their ability to adapt to high doses of drugs called tolerance by lag.

\section{Modeling}

Apart from these wet lab techniques, mathematical modeling has provided interesting insights [28,59-62] (summarized by [63]). Briefly, two main strategies can be discerned: the first one relies on estimating the switching rates between persister and non-persister growth states and assumes this process to take place continuously and stochastically (e.g. $[11,28,59,61,64])$. The balance between both switching rates 
provides a straightforward way to model a given persister level, although ignoring exactly what determines the switching rates. The second strategy focuses on the molecular mechanisms of persister formation by TA systems, with the ratio of (free) toxin over antitoxin ultimately determining, at the single-cell level, the decision to switch to the persister state (e.g. $[60,65,66,50])$. A crucial factor in this type of models is the generation of phenotypic bistability at the population level, typically requiring noisy gene expression and noise amplification through positive feedback mechanisms [67]. Both modeling strategies have their merits, and until a more integrated approach is presented, the choice between both will depend on the goal and specific focus of the study at hand.

Mathematical modeling of persistence poses several advantages. Experiments that are not feasible in the lab can be simulated to predict the outcome. It also allows to explain empirically observed persister levels in terms of the parameters encompassed by the model, and why varying some parameters has more impact on persistence than others. Consequently, evolutionary forces that shape persister levels can be identified, which should help to devise strategies to avoid high persister levels emerging in the clinic.

\section{State of the Art and Future Perspectives}

Recently, the field of microbial persistence research has exploded, as evidenced by a host of publications in top-tier journals $[10,22,23,35,48-50,54,68-70]$. Currently, it is generally accepted that persister cells are present in a bacterial population preceding antibiotic treatment [71]. It is postulated that their formation results from noisy gene expression [72] as was first suggested by Kim Lewis [6]. However, over the years, several stimuli have been shown to induce persistence. For example, sub-inhibitory 
concentrations of fluoroquinolones are known to induce persistence via activation of the tisAB/istR TA locus $[18,19]$. Other examples include quorum sensing molecules [73,74], carbon source transitions [70] and nutrient deprivation leading to activation of the stringent response [75]. As was earlier described for HipA [76], a recent model ascribes TA-regulated persistence to stochastic fluctuations in cellular concentrations of the alarmone (p)ppGpp. High (p)ppGpp levels activate TA loci through a regulatory cascade requiring inorganic polyphosphate and Lon protease targeting protein toxins [49]. For an elaborate discussion on the role of these mechanisms in persistence, the reader is referred to some excellent reviews on the topic [3,77-81].

Adding to the significance of these studies is the recent observation of a phenotypically distinct subpopulation of transiently drug-tolerant persisters in cancer cell populations. These cells are held responsible for "fractional killing" upon chemotherapy [82]. Cell-to-cell variations in protein levels were suggested to contribute to this phenomenon in which each round of therapy kills some but not all of the cells in a tumor [83]. There is a striking analogy between bacterial and cancer cell-derived persistence as both phenomena reflect a transiently phenotypic heterogeneity causing multi-drug tolerance and recurrence of disease symptoms upon removal of treatment [84]. Added insight into bacterial persistence may therefore impact research areas far beyond infectious disease.

\section{References}

1. Hobby GL, Meyer K, Chaffee E (1942) Observations on the mechanism of action of penicillin. Exp Biol Med 50 (2):281-285 
2. Bigger JW (1944) Treatment of staphylococcal infections with penicillin. Lancet 244:497-500

3. Lewis K (2010) Persister cells. Annu Rev Microbiol 64:357-372

4. Moyed HS, Bertrand KP (1983) hipA, a newly recognized gene of Escherichia coli $\mathrm{K}-12$ that affects frequency of persistence after inhibition of murein synthesis. $\mathrm{J}$ Bacteriol 155 (2):768-775

5. Moyed HS, Broderick SH (1986) Molecular cloning and expression of hipA, a gene of Escherichia coli K-12 that affects frequency of persistence after inhibition of murein synthesis. J Bacteriol 166 (2):399-403

6. Keren I, Shah D, Spoering A, Kaldalu N, Lewis K (2004) Specialized persister cells and the mechanism of multidrug tolerance in Escherichia coli. J Bacteriol 186 $(24): 8172-8180$

7. Correia FF, D'Onofrio A, Rejtar T, Li L, Karger BL, Makarova K, Koonin EV, Lewis K (2006) Kinase activity of overexpressed HipA is required for growth arrest and multidrug tolerance in Escherichia coli. J Bacteriol 188 (24):8360-8367

8. Hansen S, Vulic M, Min J, Yen TJ, Schumacher MA, Brennan RG, Lewis K (2012) Regulation of the Escherichia coli HipBA toxin-antitoxin system by proteolysis. PLoS One 7 (6):e39185. doi:10.1371/journal.pone.0039185

9. Kaspy I, Rotem E, Weiss N, Ronin I, Balaban NQ, Glaser G (2013) HipA-mediated antibiotic persistence via phosphorylation of the glutamyl-tRNA-synthetase. Nat Commun 4:3001. doi:10.1038/ncomms4001

10. Germain E, Castro-Roa D, Zenkin N, Gerdes K (2013) Molecular mechanism of bacterial persistence by HipA. Molecular Cell 52 (2):248-254.

doi:10.1016/j.molcel.2013.08.045 
11. Balaban NQ, Merrin J, Chait R, Kowalik L, Leibler S (2004) Bacterial persistence as a phenotypic switch. Science 305 (5690):1622-1625

12. Yamaguchi $Y$, Inouye M (2011) Regulation of growth and death in Escherichia coli by toxin-antitoxin systems. Nature Reviews Microbiology 9 (11):779-790. doi:10.1038/nrmicro2651

13. Yamaguchi $Y$, Park JH, Inouye M (2011) Toxin-antitoxin systems in bacteria and archaea. Annual Review of Genetics 45:61-79. doi:10.1146/annurev-genet-110410132412

14. Williams JJ, Hergenrother PJ (2012) Artificial activation of toxin-antitoxin systems as an antibacterial strategy. Trends in Microbiology 20 (6):291-298. doi:10.1016/j.tim.2012.02.005

15. Kim Y, Wang X, Zhang XS, Grigoriu S, Page R, Peti W, Wood TK (2010) Escherichia coli toxin/antitoxin pair MqsR/MqsA regulate toxin CspD. Environ Microbiol 12 (5):1105-1121

16. Kim Y, Wood TK (2010) Toxins Hha and CspD and small RNA regulator Hfq are involved in persister cell formation through MqsR in Escherichia coli. Biochem Biophys Res Commun 391 (1):209-213

17. Cheng HY, Soo VW, Islam S, McAnulty MJ, Benedik MJ, Wood TK (2013) Toxin GhoT of the GhoT/GhoS TA system damages the cell membrane to reduce ATP and to reduce growth under stress. Environ Microbiol 16 (6):1741-1754. doi:10.1111/1462-2920.12373

18. Dörr T, Lewis K, Vulić M (2009) SOS response induces persistence to fluoroquinolones in Escherichia coli. PLoS Genet 5 (12):e1000760 19. Dörr T, Vulić M, Lewis K (2010) Ciprofloxacin causes persister formation by inducing the TisB toxin in Escherichia coli. PLoS Biol 8 (2):e1000317 
20. Tripathi A, Dewan PC, Siddique SA, Varadarajan R (2014) MazF-induced Growth Inhibition and Persister Generation in Escherichia coli. Journal of Biological Chemistry 289 (7):4191-4205. doi:10.1074/jbc.M113.510511

21. Harrison JJ, Wade WD, Akierman S, Vacchi-Suzzi C, Stremick CA, Turner RJ, Ceri $\mathrm{H}$ (2009) The chromosomal toxin gene yafQ is a determinant of multidrug tolerance for Escherichia coli growing in a biofilm. Antimicrob Agents Chemother 53 (6):2253-2258

22. Helaine S, Cheverton AM, Watson KG, Faure LM, Matthews SA, Holden DW (2014) Internalization of Salmonella by macrophages induces formation of nonreplicating persisters. Science 343 (6167):204-208. doi:10.1126/science.1244705 23. Maisonneuve E, Shakespeare LJ, Jørgensen MG, Gerdes K (2011) Bacterial persistence by RNA endonucleases. Proceedings of the National Academy of Sciences of the United States of America 108 (32):13206-13211.

doi:10.1073/pnas. 1100186108

24. Fauvart M, De Groote VN, Michiels J (2011) Role of persister cells in chronic infections: clinical relevance and perspectives on anti-persister therapies. Journal of Medical Microbiology 60 (Pt 6):699-709

25. Lewis K (2001) Riddle of biofilm resistance. Antimicrob Agents Chemother 45 (4):999-1007. doi:10.1128/AAC.45.4.999-1007.2001

26. Spoering AL, Lewis K (2001) Biofilms and planktonic cells of Pseudomonas aeruginosa have similar resistance to killing by antimicrobials. J Bacteriol 183 (23):6746-6751. doi:10.1128/JB.183.23.6746-6751.2001

27. Costerton JW, Stewart PS, Greenberg EP (1999) Bacterial biofilms: a common cause of persistent infections. Science 284 (5418):1318-1322 
28. Levin BR, Rozen DE (2006) Non-inherited antibiotic resistance. Nat Rev Microbiol 4 (7):556-562

29. LaFleur MD, Qi Q, Lewis K (2010) Patients with long-term oral carriage harbor high-persister mutants of Candida albicans. Antimicrob Agents Chemother 54 (1):3944

30. Mulcahy LR, Burns JL, Lory S, Lewis K (2010) Emergence of Pseudomonas aeruginosa strains producing high levels of persister cells in patients with cystic fibrosis. J Bacteriol 192 (23):6191-6199

31. Cohen NR, Lobritz MA, Collins JJ (2013) Microbial persistence and the road to drug resistance. Cell Host Microbe 13 (6):632-642. doi:10.1016/j.chom.2013.05.009 32. Fu Y, Zhu M, Xing J (2010) Resonant activation: a strategy against bacterial persistence. Phys Biol 7 (1):16013

33. Niepa TH, Gilbert JL, Ren D (2012) Controlling Pseudomonas aeruginosa persister cells by weak electrochemical currents and synergistic effects with tobramycin. Biomaterials 33 (30):7356-7365. doi:10.1016/j.biomaterials.2012.06.092 34. Manuel J, Zhanel GG, de Kievit T (2010) Cadaverine suppresses persistence to carboxypenicillins in Pseudomonas aeruginosa PAO1. Antimicrobial Agents and Chemotherapy 54 (12):5173-5179. doi:10.1128/aac.01751-09

35. Allison KR, Brynildsen MP, Collins JJ (2011) Metabolite-enabled eradication of bacterial persisters by aminoglycosides. Nature 473 (7346):216-220.

doi:10.1038/nature10069

36. Barraud N, Buson A, Jarolimek W, Rice SA (2013) Mannitol enhances antibiotic sensitivity of persister bacteria in Pseudomonas aeruginosa biofilms. PLoS One 8 (12):e84220. doi:10.1371/journal.pone.0084220 
37. Bahar AA, Ren D (2013) Antimicrobial peptides. Pharmaceuticals (Basel) 6 (12):1543-1575. doi:10.3390/ph6121543

38. Pan J, Bahar AA, Syed H, Ren D (2012) Reverting antibiotic tolerance of Pseudomonas aeruginosa PAO1 persister cells by (Z)-4-bromo-5-(bromomethylene)3-methylfuran-2(5H)-one. PLoS One 7 (9):e45778.

doi:10.1371/journal.pone. 0045778

39. Pan J, Ren D (2013) Structural effects on persister control by brominated furanones. Bioorganic \& Medicinal Chemistry Letters 23 (24):6559-6562.

doi:10.1016/j.bmcl.2013.10.070

40. Pan J, Song F, Ren D (2013) Controlling persister cells of Pseudomonas aeruginosa PDO300 by (Z)-4-bromo-5-(bromomethylene)-3-methylfuran-2(5H)-one. Bioorganic \& Medicinal Chemistry Letters 23 (16):4648-4651.

doi:10.1016/j.bmcl.2013.06.011

41. Pan J, Xie X, Tian W, Bahar AA, Lin N, Song F, An J, Ren D (2013) (Z)-4-bromo5-(bromomethylene)-3-methylfuran-2(5H)-one sensitizes Escherichia coli persister cells to antibiotics. Applied Microbiology and Biotechnology 97 (20):9145-9154. doi:10.1007/s00253-013-5185-2

42. Conlon BP, Nakayasu ES, Fleck LE, LaFleur MD, Isabella VM, Coleman K, Leonard SN, Smith RD, Adkins JN, Lewis K (2013) Activated ClpP kills persisters and eradicates a chronic biofilm infection. Nature 503 (7476):365-370.

doi:10.1038/nature12790

43. Lewis K (2008) Multidrug tolerance of biofilms and persister cells. Curr Top Microbiol Immunol 322:107-131 
44. Hansen S, Lewis K, Vulić M (2008) Role of global regulators and nucleotide metabolism in antibiotic tolerance in Escherichia coli. Antimicrob Agents Chemother $52(8): 2718-2726$

45. De Groote VN, Verstraeten N, Fauvart M, Kint Cl, Cornelis P, Michiels J (2009) Identification of novel persistence genes in Pseudomonas aeruginosa in the combat against emerging antimicrobial resistance. Commun Agric Appl Biol Sci 74 (4):51-56 46. Spoering AL, Vulić M, Lewis K (2006) GlpD and PlsB participate in persister cell formation in Escherichia coli. J Bacteriol 188 (14):5136-5144

47. Gefen O, Gabay C, Mumcuoglu M, Engel G, Balaban NQ (2008) Single-cell protein induction dynamics reveals a period of vulnerability to antibiotics in persister bacteria. Proc Natl Acad Sci U S A 105 (16):6145-6149

48. Vega NM, Allison KR, Khalil AS, Collins JJ (2012) Signaling-mediated bacterial persister formation. Nature Chemical Biology 8 (5):431-433.

doi:10.1038/nchembio.915

49. Maisonneuve E, Castro-Camargo M, Gerdes K (2013) (p)ppGpp controls bacterial persistence by stochastic induction of toxin-antitoxin activity. Cell 154 (5):1140-1150. doi:10.1016/j.cell.2013.07.048

50. Rotem E, Loinger A, Ronin I, Levin-Reisman I, Gabay C, Shoresh N, Biham O, Balaban NQ (2010) Regulation of phenotypic variability by a threshold-based mechanism underlies bacterial persistence. Proc Natl Acad Sci U S A 107 (28):12541-12546

51. Jõers A, Kaldalu N, Tenson T (2010) The frequency of persisters in Escherichia coli reflects the kinetics of wake-up from dormancy. J Bacteriol 192 (13):3379-3384 
52. Orman MA, Brynildsen MP (2013) Dormancy is not necessary or sufficient for bacterial persistence. Antimicrobial Agents and Chemotherapy 57 (7):3230-3239. doi:10.1128/aac.00243-13

53. Helaine S, Thompson JA, Watson KG, Liu M, Boyle C, Holden DW (2010) Dynamics of intracellular bacterial replication at the single cell level. Proc Natl Acad Sci U S A 107 (8):3746-3751

54. Claudi B, Sprote P, Chirkova A, Personnic N, Zankl J, Schurmann N, Schmidt A, Bumann D (2014) Phenotypic variation of Salmonella in host tissues delays eradication by antimicrobial chemotherapy. Cell 158 (4):722-733.

doi:10.1016/j.cell.2014.06.045

55. Keren I, Minami S, Rubin E, Lewis K (2011) Characterization and transcriptome analysis of Mycobacterium tuberculosis persisters. MBio 2 (3):e00100-00111. doi:10.1128/mBio.00100-11

56. Shah D, Zhang Z, Khodursky A, Kaldalu N, Kurg K, Lewis K (2006) Persisters: a distinct physiological state of E. coli. BMC Microbiol 6:53

57. Fridman O, Goldberg A, Ronin I, Shoresh N, Balaban NQ (2014) Optimization of lag time underlies antibiotic tolerance in evolved bacterial populations. Nature [Epub ahead of print]:doi: 10.1038/nature13469. doi:10.1038/nature13469

58. Levin-Reisman I, Gefen O, Fridman O, Ronin I, Shwa D, Sheftel H, Balaban NQ (2010) Automated imaging with ScanLag reveals previously undetectable bacterial growth phenotypes. Nat Methods 7 (9):737-739. doi:10.1038/nmeth.1485 59. Kussell E, Kishony R, Balaban NQ, Leibler S (2005) Bacterial persistence: a model of survival in changing environments. Genetics 169 (4):1807-1814 60. Cogan NG (2007) Incorporating toxin hypothesis into a mathematical model of persister formation and dynamics. J Theor Biol 248 (2):340-349 
61. Gardner A, West SA, Griffin AS (2007) Is bacterial persistence a social trait? PLoS One 2 (8):e752. doi:10.1371/journal.pone.0000752

62. Klapper I, Gilbert P, Ayati BP, Dockery J, Stewart PS (2007) Senescence can explain microbial persistence. Microbiology 153 (Pt 11):3623-3630

63. Gefen O, Balaban NQ (2009) The importance of being persistent: heterogeneity of bacterial populations under antibiotic stress. FEMS microbiology reviews 33 (4):704-717. doi:10.1111/j.1574-6976.2008.00156.x

64. Hemsley CM, Luo JX, Andreae CA, Butler CS, Soyer OS, Titball RW (2014) Bacterial drug tolerance under clinical conditions is governed by anaerobic adaptation but not anaerobic respiration. Antimicrob Agents Chemother 58 (10):5775-5783. doi:10.1128/AAC.02793-14

65. Gelens L, Hill L, Vandervelde A, Danckaert J, Loris R (2013) A general model for toxin-antitoxin module dynamics can explain persister cell formation in E. coli. PLoS computational biology 9 (8):e1003190. doi:10.1371/journal.pcbi.1003190 66. Lou C, Li Z, Ouyang Q (2008) A molecular model for persister in E. coli. J Theor Biol 255 (2):205-209. doi:10.1016/j.jtbi.2008.07.035

67. Veening JW, Smits WK, Kuipers OP (2008) Bistability, epigenetics, and bethedging in bacteria. Annu Rev Microbiol 62:193-210.

doi:10.1146/annurev.micro.62.081307.163002

68. Nguyen D, Joshi-Datar A, Lepine F, Bauerle E, Olakanmi O, Beer K, McKay G, Siehnel R, Schafhauser J, Wang Y, Britigan BE, Singh PK (2011) Active starvation responses mediate antibiotic tolerance in biofilms and nutrient-limited bacteria. Science 334 (6058):982-986. doi:10.1126/science.1211037 
69. Wakamoto Y, Dhar N, Chait R, Schneider K, Signorino-Gelo F, Leibler S, McKinney JD (2013) Dynamic persistence of antibiotic-stressed mycobacteria. Science 339 (6115):91-95. doi:10.1126/science.1229858

70. Amato SM, Orman MA, Brynildsen MP (2013) Metabolic control of persister formation in Escherichia coli. Molecular Cell 50 (4):475-487.

doi:10.1016/j.molcel.2013.04.002

71. Keren I, Kaldalu N, Spoering A, Wang Y, Lewis K (2004) Persister cells and tolerance to antimicrobials. FEMS Microbiol Lett 230 (1):13-18

72. Fraser D, Kærn M (2009) A chance at survival: gene expression noise and phenotypic diversification strategies. Mol Microbiol 71 (6):1333-1340

73. Möker N, Dean CR, Tao J (2010) Pseudomonas aeruginosa increases formation of multidrug-tolerant persister cells in response to quorum-sensing signaling molecules. J Bacteriol 192 (7):1946-1955

74. Leung V, Levesque CM (2012) A stress-inducible quorum-sensing peptide mediates the formation of persister cells with noninherited multidrug tolerance. Journal of Bacteriology 194 (9):2265-2274. doi:10.1128/jb.06707-11

75. Gao W, Chua K, Davies JK, Newton HJ, Seemann T, Harrison PF, Holmes NE, Rhee HW, Hong JI, Hartland EL, Stinear TP, Howden BP (2010) Two novel point mutations in clinical Staphylococcus aureus reduce linezolid susceptibility and switch on the stringent response to promote persistent infection. PLoS Pathogens 6 (6):e1000944. doi:10.1371/journal.ppat.1000944

76. Korch SB, Henderson TA, Hill TM (2003) Characterization of the hipA7 allele of Escherichia coli and evidence that high persistence is governed by (p)ppGpp synthesis. Mol Microbiol 50 (4):1199-1213 
77. Helaine S, Kugelberg E (2014) Bacterial persisters: formation, eradication, and experimental systems. Trends Microbiol 22 (7):417-424.

doi:10.1016/j.tim.2014.03.008

78. Maisonneuve E, Gerdes K (2014) Molecular mechanisms underlying bacterial persisters. Cell 157 (3):539-548. doi:10.1016/j.cell.2014.02.050

79. Amato SM, Fazen CH, Henry TC, Mok WW, Orman MA, Sandvik EL, Volzing KG, Brynildsen MP (2014) The role of metabolism in bacterial persistence. Frontiers in microbiology 5:70. doi:10.3389/fmicb.2014.00070

80. Prax M, Bertram R (2014) Metabolic aspects of bacterial persisters. Frontiers in cellular and infection microbiology 4:148. doi:10.3389/fcimb.2014.00148 81. Kint Cl, Verstraeten N, Fauvart M, Michiels J (2012) New-found fundamentals of bacterial persistence. Trends in Microbiology 20 (12):577-585. doi:10.1016/j.tim.2012.08.009

82. Sharma SV, Lee DY, Li B, Quinlan MP, Takahashi F, Maheswaran S, McDermott U, Azizian N, Zou L, Fischbach MA, Wong KK, Brandstetter K, Wittner B, Ramaswamy S, Classon M, Settleman J (2010) A chromatin-mediated reversible drug-tolerant state in cancer cell subpopulations. Cell 141 (1):69-80 83. Spencer SL, Gaudet S, Albeck JG, Burke JM, Sorger PK (2009) Non-genetic origins of cell-to-cell variability in TRAIL-induced apoptosis. Nature 459 (7245):428432

84. Glickman MS, Sawyers CL (2012) Converting cancer therapies into cures: lessons from infectious diseases. Cell 148 (6):1089-1098.

doi:10.1016/j.cell.2012.02.015 


\section{Figure captions}

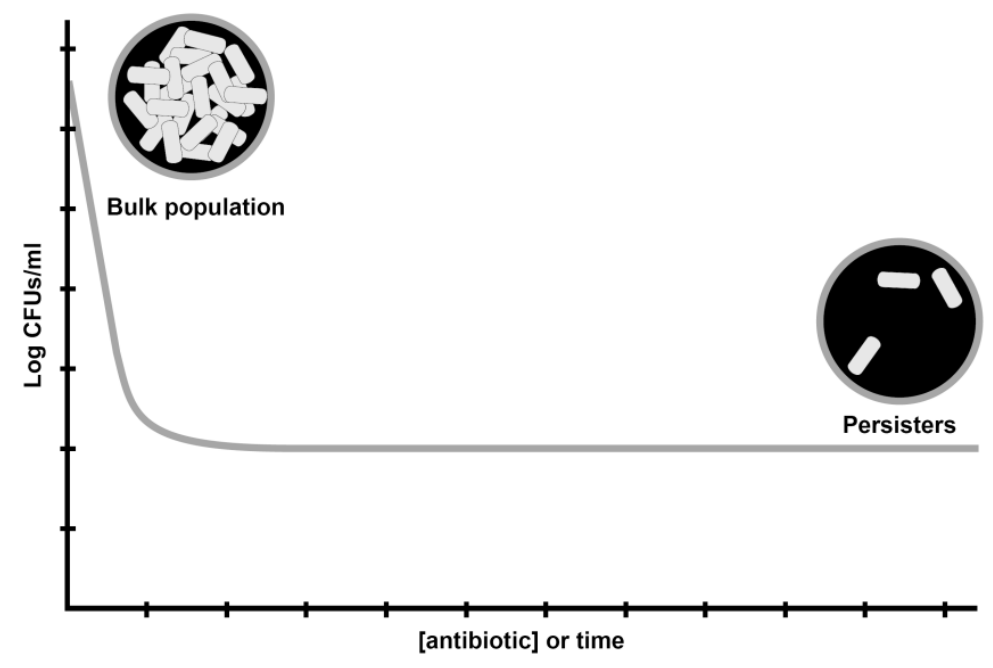

Figure 1: Illustration of persistence. The majority of cells in a bacterial culture is efficiently killed by relatively low concentrations of antibiotics. However, beyond a certain threshold, a killing plateau is observed as only persister cells remain viable. When regrown in fresh medium, the surviving cells generate a population as sensitive to the antibiotic as the original population. 